\title{
Two-dimensional computational modeling of thin film evaporation
}

\author{
Yiğit Akkuş ${ }^{\mathrm{a}}$, Hakan I. Tarman ${ }^{\mathrm{b}}$, Barbaros Çetin ${ }^{\mathrm{c},}{ }^{*}$, Zafer Dursunkaya ${ }^{\mathrm{b}}$ \\ a ASELSAN A.S., 06172, Yenimahalle, Ankara, Turkey \\ b Dept. of Mechanical Eng., Middle East Technical University, 06800 Çankaya, Ankara, Turkey \\ ${ }^{\mathrm{c}}$ Microfluidics \& Lab-on-a-chip Research Group, Mech. Eng. Dept., İ.D. Bilkent University, 06800 Ankara, Turkey
}

\section{A R T I C L E I N F O}

\section{Article history:}

Received 15 January 2017

Received in revised form

9 May 2017

Accepted 17 July 2017

\section{Keywords:}

Thin film evaporation

Evaporating meniscus

Lubrication theory

\begin{abstract}
A B S T R A C T
A considerable amount of the evaporation originates from the close vicinity the three-phase contact line in an evaporating extended meniscus due to the low thermal resistance across the ultra thin film. Evaporation taking place within the thin film region is commonly modeled using the uni-directional flow assumption of the liquid following the lubrication approximation. Although the uni-directional flow based models may yield practically reasonable results in terms of the cumulative quantities such as total evaporation rate, the underlying physics of the problem cannot be explained solely by uni-directional flow, especially when the dominant transverse liquid motion is considered near the close proximity of the contact line. The present study develops a solution methodology to enable the solution of steady, incompressible, 2-D conservation of mass and linear momentum equations for the liquid flow in an evaporating thin film. Solution methodology includes the coupling of an uni-directional solver with high precision numerics, a higher order bi-directional spectral element solver and a finite element solver. The novelty of the present study is that steady, 2-D conservation of mass and linear momentum equations are considered in the modeling of thin film evaporation without the exclusion of any terms in the conservation equations.
\end{abstract}

() 2017 Elsevier Masson SAS. All rights reserved.

\section{Introduction}

Thin film evaporation is the central issue in various natural and technological processes such as perspiration, micro-electronics cooling, nucleate boiling and self-assembly operations [1]. The adjoining region near the contact line where liquid, vapor and solid phases meet, has the maximum evaporation rates within the extended meniscus due to its small thermal resistance.

Three-phase contact line may be present in different geometries such as liquid meniscus in a container, drop of liquid or vapor bubble on a solid substrate [2]. Heat pipes or vapor chambers are the most common examples in which evaporation from liquid meniscus takes place. Liquid is steadily supplied to the evaporating meniscus from the condenser which enables the construction of these self-regulating devices. Evaporation from a drop of liquid, on the other hand, is present when a solid surface is cooled by droplet deposition. Another presence of evaporation from a thin film is seen at intersection of a bubble with a hot solid substrate where thin film forms between solid and the gas-liquid interface.

\footnotetext{
* Corresponding author.

E-mail address: barbaros.cetin@bilkent.edu.tr (B. Çetin).
}

Modeling of evaporation requires the application of simplifications due to the inherent complexity of the problem. Lubrication theory approximation, which assumes the uni-directional liquid flow, is a common tool to model the liquid flow in evaporating thin films [3-19]. Based on lubrication theory, different physical effects can be investigated. The effect of thermocapillarity increases with elevated superheats and is considered in many studies [20,21]. For certain types of fluids, acute errors can be made by neglecting the thermocapillary effect $[22,23]$. The effects of slip boundary condition $[24,25]$ and liquid polarity [26] were also investigated in previous studies.

Evaporation modeling with an extended version of the lubrication theory also exists in the literature [27]. Extended version uses a domain perturbation method to develop the higher-order solution in terms of series expansion about lubrication condition. Zeroth and first-order closed-form solutions are taken into consideration. Zeroth-order closed-form reduces formulation to the lubrication theory. First-order closed-form adds the inertial terms of the longitudinal momentum into formulation.

When conservation of mass, linear momentum and energy equations are subjected to an order of magnitude analysis depending on the scaling of the thickness of the absorbed layer as 


\begin{tabular}{ll} 
Nomenclature \\
\\
Symbols \\
$A$ & Dispersion constant, $\mathrm{J}$ \\
$A_{r}$ & Dispersion constant for retarded films, J \\
$c_{p}$ & Specific heat capacity, $\mathrm{J} / \mathrm{kg} \cdot \mathrm{K}$ \\
$h_{l v}$ & Latent heat of evaporation, $\mathrm{J} / \mathrm{kg}$ \\
$k$ & Thermal conductivity, $\mathrm{W} / \mathrm{m} \cdot \mathrm{K}$ \\
$\ell^{*}$ & Extent of the evaporation $\mathrm{zone}, \mathrm{m}$ \\
$M$ & Molar mass of liquid, $\mathrm{kg} / \mathrm{mol}$ \\
$\dot{m}$ & Mass flow rate, $\mathrm{kg} / \mathrm{s}$ \\
$\dot{m}^{\prime}$ & Mass flow rate per unit length, $\mathrm{kg} / \mathrm{m} \cdot \mathrm{s}$ \\
$\dot{m}^{\prime \prime}$ & Mass flux, $\mathrm{kg} / \mathrm{m}^{2} \cdot \mathrm{s}$ \\
$\mathbf{n}$ & Unit normal vector \\
$p$ & Pressure, Pa \\
$q^{\prime \prime}$ & Heat flux, $\mathrm{W} / \mathrm{m}^{2}$ \\
$R$ & Radius of curvature of liquid-vapor interface, $\mathrm{m}$ \\
$R_{u}$ & Universal gas constant, $\mathrm{J} / \mathrm{mol} \cdot \mathrm{K}$ \\
$s$ & Transformed (standard) longitudinal coordinate, $\mathrm{m}$ \\
$t$ & Transformed (standard) transversal coordinate, $\mathrm{m}$ \\
$\mathbf{t}$ & Unit tangential vector \\
$T$ & Temperature, $\mathrm{K}$ \\
$\mathbf{u}$ & Velocity vector, $\mathrm{m} / \mathrm{s}$ \\
$u$ & Longitudinal velocity, $\mathrm{m} / \mathrm{s}$ \\
$v$ & Transversal velocity, $\mathrm{m} / \mathrm{s}$ \\
$v_{g}$ & Specific volume of gas, $\mathrm{m}^{3} / \mathrm{kg}$ \\
$v_{l}$ & Specific volume of liquid, $\mathrm{m}^{3} / \mathrm{kg}$ \\
\hline
\end{tabular}

$\begin{array}{ll}V & \text { Molar volume, } \mathrm{m}^{3} / \mathrm{mol} \\ x & \text { Longitudinal coordinate, } \mathrm{m} \\ y & \text { Transversal coordinate, } \mathrm{m}\end{array}$

Greek symbols

$\begin{array}{ll}\delta & \text { Liquid film thickness, } \mathrm{m} \\ \delta^{*} & \text { Contact line thickness, } \mathrm{m} \\ \mu & \text { Dynamic viscosity, } \mathrm{kg} / \mathrm{m} \cdot \mathrm{s} \\ \nu & \text { Kinematic viscosity, } \mathrm{m}^{2} / \mathrm{s} \\ \Omega & \text { Problem domain, } \mathrm{m}^{2} \\ \rho & \text { Density, } \mathrm{kg} / \mathrm{m}^{3} \\ \sigma & \text { Surface tension, } \mathrm{N} / \mathrm{m} \\ \overline{\bar{\sigma}} & \text { Stress tensor, Pa } \\ \boldsymbol{\sigma}^{\prime} & \text { Deviatoric stress tensor, Pa } \\ \hat{\sigma} & \text { Accommodation coefficient } \\ \theta & \text { Apparent contact angle, }{ }^{\circ}\end{array}$

\section{Subscripts}

$0 \quad$ inlet of the problem domain

c capillary

d disjoining

evap evaporation

$l \quad$ liquid

lv liquid-vapor

st standard

$v \quad$ vapor

$v, l v \quad$ vapor just above the liquid-vapor interface

w wall the transversal characteristic length and entire length of the thin film region as longitudinal characteristic length, governing equations are reduced to the classic lubrication formulation $[27,28]$. The scaling analysis yields a much smaller value than unity for the ratio of transverse velocity to longitudinal velocity. Therefore, unidirectional liquid flow is assumed within the thin film region. However, in the close vicinity of the contact line, where the local rate of evaporation is at its peak value, transverse velocity dominates the longitudinal one. To capture the physics of the evaporation problem within the entire domain, bi-directional liquid flow needs to be considered.

The main objective of the current study is to propose a general thin film evaporation model based on bi-directional liquid flow. The solution scheme includes an iteration process which consists of successive implementation of three coupled solvers; unidirectional solver with high precision numerics, higher order bidirectional spectral element solver and finite element solver. The novel contribution of the present study is that steady, 2-D conservation of mass and linear momentum equations for the liquid flow within the thin film are solved to model the evaporation without neglecting any terms.

\section{Problem description}

A steadily evaporating two-dimensional extended meniscus on a hypothetical perfectly flat heated surface, as illustrated in Fig. 1, is investigated.

Evaporating thin film region is characterized by high evaporation rates due to low thermal resistance of thin film, whereas intrinsic (bulk) meniscus region has high resistance to heat conduction due to thicker film. At the contact line, evaporation is suppressed by dispersion force originating from the solid-liquid molecular interactions. Steady, laminar, incompressible flow of Newtonian, spreading and non-polar liquid is provided from intrinsic meniscus to evaporating thin film region to replace the evaporating liquid in the close vicinity of the contact line. Assuming a sufficiently small Bond number, the effect of gravity is neglected. The heated solid substrate under the liquid film is assumed to have a constant wall temperature. Liquid is assumed to evaporate into its saturated vapor phase which has uniform physical properties. Variation of physical properties with the temperature is neglected within the solution domain. Zero shear stress is assumed at the interface. For the normal stress balance, the effects of capillary and disjoining pressures are considered [29]. Applying these assumptions, two-dimensional conservation of mass, linear momentum and energy equations are as follows:

$\partial_{x} u+\partial_{y} v=0$

$\rho\left(u \partial_{x} u+v \partial_{y} u\right)=-\partial_{x} p+\mu\left(\partial_{x x} u+\partial_{y y} u\right)$

$\rho\left(u \partial_{x} v+v \partial_{y} v\right)=-\partial_{y} p+\mu\left(\partial_{x x} v+\partial_{y y} v\right)$

$\rho c_{p}\left(u \partial_{x} T+v \partial_{y} T\right)=k\left(\partial_{x x} T+\partial_{y y} T\right)$.

The associated boundary conditions with Equations (1a)-(1d) are specified as follows:

$u=0, \quad v=0, \quad T=T_{w} \quad$ at $\quad y=0$, 


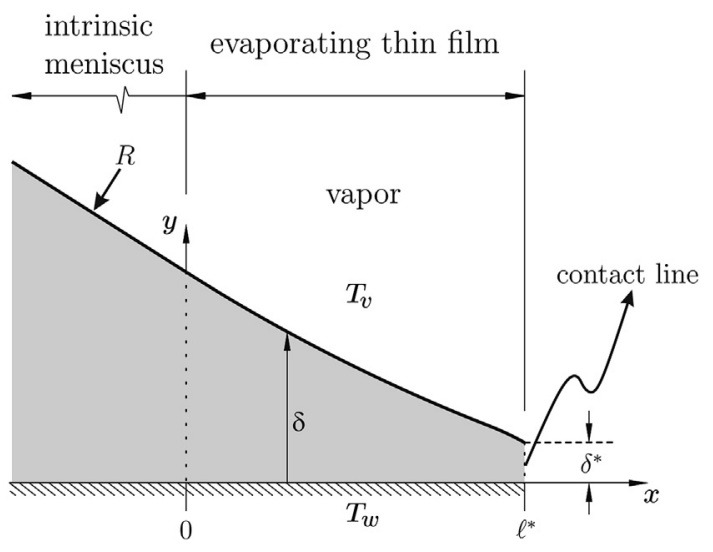

Fig. 1. Physical domain.

$\mathbf{n} \cdot \boldsymbol{\sigma}^{\prime} \cdot \mathbf{t}=0, \quad-p+\mathbf{n} \cdot \boldsymbol{\sigma}^{\prime} \cdot \mathbf{n}+p_{v}=p_{c}+p_{d}, \quad T=T_{l v}(\boldsymbol{x}) \quad$ at $\quad y$ $=\delta$,

$u=u_{0}(y), \quad v=v_{0}(y) \quad$ at $\quad x=0$,

$u=0, \quad v=0 \quad$ at $\quad x=\ell^{*}$,

where $T_{w}, T_{v}$ and $T_{l v}$ are the wall, bulk vapor and interface temperatures, respectively. $\ell^{*}$ is the extent of the evaporation zone. $\sigma^{\prime}$ is the deviatoric stress defined as

$\boldsymbol{\sigma}^{\prime}=\mu\left(\begin{array}{cc}2 \partial_{x} u & \partial_{x} v+\partial_{y} u \\ \partial_{y} u+\partial_{x} v & 2 \partial_{y} v\end{array}\right)$

$p$ and $p_{v}$ are the liquid and bulk vapor pressures, respectively. $p_{c}$ is the capillary pressure given by the relation

$p_{c}=\sigma \frac{d^{2} \delta / d x^{2}}{\left[1+(d \delta / d x)^{2}\right]^{3 / 2}}$

and $p_{d}$ is the disjoining pressure. For completely wetting systems of non-polar liquids, disjoining pressure is expressed as the inverse powers of film thickness [30]:

$p_{d}=\frac{A}{\delta^{3}}, \quad \delta \leq 20 \mathrm{~nm}$,

$p_{d}=\frac{A_{r}}{\delta^{4}}, \quad \delta \geq 40 \mathrm{~nm}$,

where $A$ is the dispersion constant and $A_{r}$ is the dispersion constant for retarded films.

In the described problem, governing equations and their boundary conditions are functions of film thickness which is unknown a priori. The extent of the evaporation zone, $\ell^{*}$, and the film thickness at the contact line, $\delta^{*}$, are also unknowns, whose values are obtained from the solution of the problem. Therefore, the geometry of the problem domain should be considered as dynamic. Consequently, afore-declared formulae are not sufficient for the solution of the problem. Moreover, evaporative mass flux at the interface must be associated to the liquid flow. Therefore, a constitutive equation must be added to the governing equations for the closure of the problem, which is the Kucherov-Rikenglaz [31] equation. This formulation, written below, defines the evaporative mass flux at the interface and it is commonly attributed to Schrage [32] in the literature.

$\dot{m}^{\prime \prime}$ evap $=\frac{2 \widehat{\sigma}}{2-\widehat{\sigma}}\left(\frac{M}{2 \pi R_{u}}\right)^{1 / 2}\left(\frac{p_{v, l v}}{T_{l v}^{1 / 2}}-\frac{p_{v}}{T_{v}^{1 / 2}}\right)$.

In the study published in 1976, Wayner et al. [3] suggested the following relation assuming small superheat values:

$T_{l v}^{1 / 2} \approx T_{v}^{1 / 2}$

With this simplification, evaporative mass flux at the interface reduces to a function of pressure difference between bulk vapor phase and the vapor just above the interface,

$\dot{m}_{\text {evap }}^{\prime \prime}=\frac{2 \widehat{\sigma}}{2-\widehat{\sigma}}\left(\frac{M}{2 \pi R_{u} T_{v}}\right)^{1 / 2}\left(p_{v, l v}-p_{v}\right)$

The equilibrium pressure difference at the liquid-vapor interface is related to the temperature difference between phases, the Clapeyron effect; and surface forces at the interface, the curvature effect. The relationship between equilibrium temperature and vapor pressure is obtained from the Clasius-Clapeyron equation. The surface forces which determine the shape of the interface, are capillary and disjoining forces. Modified Kelvin equation is applied to relate these forces to the pressure jump at the interface. Details of the derivation of the terms originating from Clapeyron and curvature effects are presented in Appendix A. The equilibrium pressure difference at the liquid-vapor interface can be formulated as the combination of afore-mentioned effects:

$p_{v, l v}-p_{v}=\frac{h_{l v} M p_{v}}{R_{u} T_{l v} T_{v}}\left(T_{l v}-T_{v}\right)-\frac{V_{l} p_{v}}{R_{u} T_{l v}}\left(p_{c}+p_{d}\right)$.

When Equation (9) is substituted to Equation (8), the following formula by Sujanani and Wayner [4], is obtained.

$\dot{m}_{\text {evap }}^{\prime \prime}=a\left(T_{l v}-T_{v}\right)-b\left(p_{c}+p_{d}\right)$,

where

$a=\frac{2 \widehat{\sigma}}{2-\widehat{\sigma}}\left(\frac{M}{2 \pi R_{u} T_{l v}}\right)^{1 / 2}\left(\frac{M p_{v} h_{l v}}{R_{u} T_{v} T_{l v}}\right)$

$b=\frac{2 \widehat{\sigma}}{2-\widehat{\sigma}}\left(\frac{M}{2 \pi R_{u} T_{l v}}\right)^{1 / 2}\left(\frac{p_{v} V_{l}}{R_{u} T_{l v}}\right)$.

\section{Theoretical modeling}

Lubrication approximation neglects inertial and longitudinal diffusive terms of the $x$-momentum equation, Equation (1b), and all terms, except the pressure gradient, of the $y$-momentum equation, Equation (1c). Unlike many studies which utilize the lubrication approximation for the modeling of thin film evaporation, the present study aims to solve the afore-mentioned governing equations without omitting any terms.

In what follows, different modeling efforts are presented. In the solution of the evaporation problem, these models are going to be used as successive steps. 


\subsection{Uni-directional modeling}

Following an order of magnitude analysis and keeping the leading order problem, governing equations can be written as $[27,28]$ :

$\partial_{x} u+\partial_{y} v=0$,

$0=-\partial_{x} p+\mu \partial_{y y} u$

$0=-\partial_{y} p$

$0=\partial_{y y} T$

and the associated boundary conditions are:

$u=0, \quad v=0, \quad T=T_{w} \quad$ at $\quad y=0$,

$\partial_{y} u=0, \quad-p+p_{v}=p_{c}+p_{d}, \quad T=T_{l v}(x) \quad$ at $\quad y=\delta$,

$u=u_{0}(y), \quad v=v_{0}(y)$ at $\quad x=0$,

$u=0, \quad v=0 \quad$ at $\quad x=\ell^{*}$.

The solution of Equations (12b) and (12c) with the boundary conditions given in Equations (13a) and (13b) yields a longitudinal velocity distribution as:

$u=\frac{1}{\mu} d_{x} p\left(\frac{y^{2}}{2}-\delta y\right)$

Note that Equation (12c) suggests the variation of liquid pressure being only in the longitudinal direction. Consequently, the pressure balance given in Equation (13b) is actually valid not only at the interface but also along the transverse direction. Therefore, this pressure balance, generally known as the augmented YoungLaplace equation, can be written as a separate equation for the entire domain, from which liquid pressure or its gradient can be obtained. When it is substituted into Equation (14), the longitudinal velocity distribution has the following form:

$u=-\frac{1}{\mu} d_{x}\left(p_{c}+p_{d}\right)\left(\frac{y^{2}}{2}-\delta y\right)$

The longitudinal velocity can be integrated over the transverse direction to obtain the mass flow rate of the liquid as:

$\dot{m}_{l}^{\prime}=-\frac{\delta^{3}}{3 \nu} d_{x}\left(p_{c}+p_{d}\right)$

The order of magnitude analysis which results in Equation (12d) shows that the convection of heat in the longitudinal direction is negligible compared to the conduction along transverse direction due to the extremely small flow velocities and film thickness. Therefore, temperature distribution can be derived with the thermal boundary conditions, Equations (13a) and (13b), as:

$T=\frac{T_{l v}-T_{w}}{\delta} y+T_{w}$

Evaporative mass flux is linked to heat flux through the latent heat of vaporization. Following the balance between conductive heat flux and mass flux in conjunction with the lubrication approximation, the interface temperature can be written as:
$T_{l v}=T_{w}-\frac{h_{l v} \dot{m}^{\prime \prime}{ }_{\text {evap }} \delta}{k}$.

Combining Equations (10) and (18), the evaporative mass flux can be deduced as [28]:

$$
\dot{m}_{\text {evap }}^{\prime \prime}=\frac{1}{1+a \delta h_{l v} / k}\left[a\left(T_{w}-T_{v}\right)-b\left(p_{c}+p_{d}\right)\right] .
$$

Change of liquid mass flow rate in the direction of flow is caused by the evaporative mass flux to the vapor phase, resulting a mass balance in the following form:

$\frac{d \dot{m}_{l}^{\prime}}{d x}=-\dot{m}_{\text {evap }}^{\prime \prime}$

that is

$\frac{1}{3 \nu} d_{x}\left[\delta^{3} d_{x}\left(p_{c}+p_{d}\right)\right]=\frac{1}{1+a \delta h_{l v} / k}\left[a\left(T_{w}-T_{v}\right)-b\left(p_{c}+p_{d}\right)\right]$.

Equation (21) is a $4^{\text {th }}$ order, non-linear differential equation of the film thickness. This initial value problem requires four initial conditions at the beginning of the domain of integration. The first three are the film thickness, and its first and second derivatives, which are related to the contact angle, and intrinsic meniscus radius. The third derivative is related to the mass inflow rate of the liquid [19]. Solution of this equation yields the film thickness distribution within the problem domain.

\subsection{Bi-directional SEM modeling}

As long as boundary of the domain is specified, continuity and Navier Stokes equations can be solved using one of the regular computational fluid dynamics (CFD) methods. In the evaporating thin film problems, film thickness distribution, which is one of the boundaries of the problem domain, or the extent of the evaporation zone, $\ell^{*}$, are obtained as the result of the solution. Consequently, the problem cannot be directly solved using a regular CFD technique in a single solution stage. However, if the film thickness distribution within the extent of the evaporation were provided, application of a standard CFD method would be possible. Therefore, as a part of an iterative solution scheme, an initial estimate is used for the film thickness distribution. The resultant film thickness distribution of the uni-directional model can be used as the estimate. Due to the fact that pressure gradient in the longitudinal direction and evaporative mass flux at the interface are the functions of the film thickness for the uni-directional model, their distributions are inherently specified within the problem domain. Furthermore, if the distribution of pressure gradient along the transverse direction were available before solving the system of equations, longitudinal and transverse velocities could be solved from the momentum equations. Therefore, an initial estimate is also made for the distribution of the pressure gradient along the transverse direction, within a suitable iterative solution scheme. The resultant system of equation has the following form:

$\rho\left(u \partial_{x} u+v \partial_{y} u\right)=-\partial_{x} p+\mu\left(\partial_{x x} u+\partial_{y y} u\right)$,

$\rho\left(u \partial_{x} v+v \partial_{y} v\right)=-\partial_{y} p+\mu\left(\partial_{x x} v+\partial_{y y} v\right)$,

Due to the fact that the distribution of the liquid pressure gradient is specified by assigning estimated values, boundary conditions of Equations (22a) and (22b) are given only for velocity components: 
$u=0, \quad v=0 \quad$ at $\quad y=0$,

$\mathbf{u} \cdot \mathbf{n}=m_{\text {evap }}^{\prime \prime} / \rho$ at $y=\delta$,

$u=u_{0}, \quad v=v_{0} \quad$ at $\quad x=0$,

$u=0, \quad v=0 \quad$ at $\quad x=\ell^{*}$.

Spectral element method (SEM), which is an $h p$-formulation of the finite element method (FEM), is used in the solution of Equations (22a) and (22b). SEM uses high degree piecewise polynomials (p), thus a fast convergence to the exact solution is realized with fewer number of elements (h). With the application of the SEM, higher order, accurate and continuous velocity distribution is aimed to achieve. Furthermore, the short range in which evaporative heat flux reaches its highest point and suddenly drops to zero, can be easily solved with adequate resolution by the help of nonuniformly spaced Gauss-Lobatto-Legendre (GLL) nodes which enable high resolution near boundaries. The details of the application of SEM to Equations (22a) and (22b), and to the corresponding boundary conditions are provided in Appendix B.

\subsection{Bi-directional FEM modeling}

Once the film thickness variation is obtained, the flow geometry can be generated, and the evaporative mass flux on the top boundary can be assigned as the velocity boundary condition. To determine the pressure field, more specifically the pressure variation in the transverse direction, the incompressible form of the 2-D Navier-Stokes equations together with continuity equation can be solved numerically:

$\nabla \cdot \mathbf{u}=0$

$\rho(\mathbf{u} \cdot \nabla) \mathbf{u}=-\nabla p+\mu \nabla^{2} \mathbf{u}$

In this study, FEM-based modeling is utilized for the solution of the above system by implementing MATLAB interface of the COMSOL software. Since the output of the FEM modeling is the pressure gradient, the element of order of $\mathrm{P} 3+\mathrm{P} 2$, which uses thirdorder elements for momentum and second-order elements for continuity, is used. Once the primitive variables are solved, the pressure gradient in the transverse direction is obtained in the post-processing step at the specified GLL nodes.

\section{Computational scheme}

Different modeling approaches were presented in Section 3. In the following section, these modelings are implemented to construct an iterative computational scheme to solve the steady, 2D continuity and momentum equations for the thin film evaporation problem. Computational scheme, presented in Fig. 2, includes four basic steps in a single iteration phase. Details of each step are provided in the following parts of this chapter.

\subsection{Step-0: Initialization}

In Step-0, initial estimates for the film thickness and pressure gradient distributions in longitudinal direction are obtained from uni-directional modeling. Uni-directional modeling requires the solution of Equation (21), which is a $4^{\text {th }}$ order, non-linear differential equation for the film thickness. In the solution of Equation (21), numerical integration starts from the intrinsic meniscus region and all the boundary conditions are defined at the intrinsic meniscus without forcing any pre-assumed shape of the thin film at the contact line. Therefore, the contact line thickness is calculated as a result of the numerical analysis unlike the majority of the previous numerical studies where contact line thickness was calculated before starting the numerical integration. In addition, the uni-directional model uses quadruple precision numerics due to the fact that the relative convergence criteria within double precision numerics is insufficient to capture the rapid changes in higher derivatives occurring in the close proximity of the contact line. This usage of quadruple precision numerics enables the calculation of highly accurate higher derivatives needed in the integration process. The details of the solution approach of the unidirectional model are given in Ref. [19].

\subsection{Step-1: Bi-directional higher order flow modeling using SEM without continuity and transverse pressure gradient}

In Step-1, Equations (22a) and (22b) are solved by SEM. These equations require initial estimates for the film thickness and pressure gradient distributions. The pressure gradient in transverse direction is assumed to be zero at this step. Following the calculation of initial estimates, Equations (22a) and (22b) with the associated boundary conditions, Equations (23a)-(23d), are solved using SEM (Appendix B). The solution yields velocity field within the problem domain with high resolution. However, this solution needs to be improved by following steps in which the error in the solution of velocity distribution, originating from the lack of pressure gradient in the transverse direction and the mass continuity, is corrected.

\subsection{Step-2: Bi-directional flow modeling using FEM solver}

In Step-2, the flow and pressure fields are obtained using the film thickness variation from Step-0, through an FEM solver. In this paper, COMSOL Multiphysics is utilized as the FEM solver, and every step of the iterative algorithm is automated through the MATLAB interface of the COMSOL. The solution domain is generated by defining multiple neighboring trapezoids using the point-wise distribution of the film thickness from Step-0. On the top surface of each trapezoid, the longitudinal and transverse velocities are assigned as the boundary conditions. The pressure gradient at the GLL nodes are evaluated at the post-processing to be used as input to the SEM modeling.

\subsection{Step-3: Bi-directional higher order flow modeling using SEM without continuity}

In Step-3, the flow is solved using SEM including the effect of pressure gradient in the transverse direction. Estimation of pressure variation in $y$-direction is taken from the result of FEM solution of the previous step. SEM solution yields bi-directional velocity distribution within the problem domain, however, the results need further corrections due to the fact that continuity equation is not solved in this step.

\subsection{Step-4: Uni-directional quadruple precision evaporating contact line modeling with the effect of transverse velocity}

So far, distributions of $u$ and $v$ velocity in the thin film region have been determined without considering the conservation of mass in the spectral formulation. However, the film thickness distribution, which defines the boundary of the solution domain, has to be updated such that conservation of mass must hold for the bidirectional flow based solution. Uni-directional modeling can be 


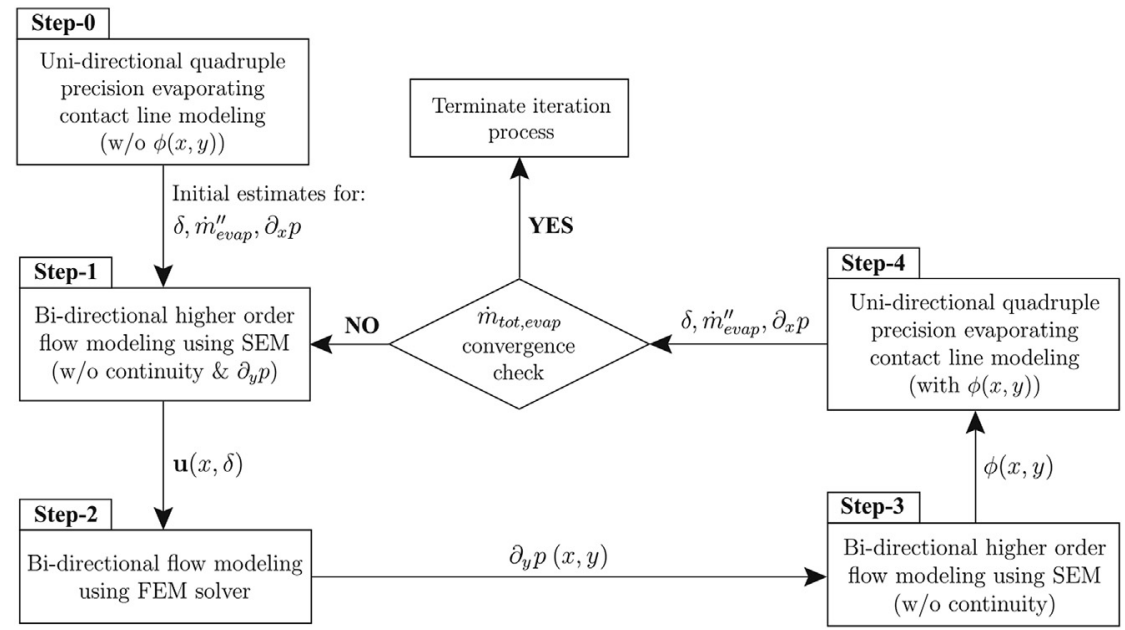

Fig. 2. Flow chart of the computational scheme.

used to obtain the mass conservation.

In the solution of the uni-directional flow, only $x$-momentum equation is considered. Furthermore, inertial and longitudinal diffusive terms are neglected due to lubrication approximation. Bidirectional flow based models, on the other hand, need to include these neglected terms. $x$-momentum equation, Equation (1b), can be written as follow:

$\partial_{y y} u=\partial_{x} p / \mu+\left(u \partial_{x} u+v \partial_{y} u\right) / \nu-\partial_{x x} u$

Furthermore, the neglected terms in the uni-directional flow model are grouped and defined as $\phi$,

$\phi(x, y) \equiv\left(u \partial_{x} u+v \partial_{y} u\right) / \nu-\partial_{x x} u$.

Then, Equation (27) can be written in terms of $\phi$ as;

$\partial_{y y} u=\partial_{x} p / \mu+\phi(x, y)$.

The term on the left side and the first term at the right hand side are the terms existing in the derivation of the evaporation models based on both uni-directional and bi-directional flow. The term $\phi(x, y)$, on the other hand, exists only in the formulation of evaporation model based on bi-directional flow.

Although the spectral element formulation of the bi-directional flow considers only the conservation of linear momentum, unidirectional flow based solution takes both mass and momentum conservation into consideration. Then, in order to satisfy conservation of mass, uni-directional flow model can be used. The term $\phi(x, y)$ contains the effect of bi-directional solution. If the unidirectional model is applied by adding the values of the term $\phi(x, y)$ into the formulation, in other words, if Equation (28) is used in formulation of uni-directional flow, a new film thickness distribution including the effect of bi-directional solution, can be achieved which satisfies the conservation of mass. However, the values of the term $\phi(x, y)$ within the domain have a two-dimensional distribution as a natural consequence of SEM. In order to use the uni-directional flow model, Equation (28) needs to be integrated in transverse direction. With the presence of the 2-D $\phi(x, y)$ term, Equation (28) cannot be integrated analytically. To overcome this difficulty, Equation (27) is integrated in transverse direction to get the average values of the term at the $x$-coordinates, $\bar{\phi}(x)$, are calculated. Integrating Equation (28) at a constant $x$-coordinate over $y$-coordinate using no slip boundary condition at the wall surface and no shear assumption at the liquid-vapor interface
Table 1

Physical data of [5].

\begin{tabular}{lll}
\hline Vapor temperature $(\mathrm{K})$ & $T_{v}$ & 300 \\
\hline Wall temperature $(\mathrm{K})$ & $T_{w}$ & 301 \\
Vapor pressure $(\mathrm{Pa})$ & $p_{v}$ & $1.06 \times 10^{6}$ \\
Density of saturated vapor $\left(\mathrm{kg} / \mathrm{m}^{3}\right)$ & $\rho_{v}$ & 9 \\
Density of liquid $\left(\mathrm{kg} / \mathrm{m}^{3}\right)$ & $\rho_{l}$ & 600 \\
Latent heat of evaporation $(\mathrm{J} / \mathrm{kg})$ & $h_{l v}$ & $1.18 \times 10^{6}$ \\
Surface tension $(\mathrm{N} / \mathrm{m})$ & $\sigma$ & $2.0 \times 10^{-2}$ \\
Dynamic viscosity of liquid $(\mathrm{Pa} \cdot \mathrm{s})$ & $\mu$ & $1.3 \times 10^{-4}$ \\
Thermal conductivity of liquid $(\mathrm{W} / \mathrm{m} \cdot \mathrm{K})$ & $k$ & 0.48 \\
Molar mass of liquid $(\mathrm{kg} / \mathrm{mol})$ & $M$ & $17.3 \times 10^{-3}$ \\
Molar volume of liquid $\left(\mathrm{m}^{3} / \mathrm{mol}\right)$ & $V_{l}$ & $28.8 \times 10^{-6}$ \\
Accommodation coefficient & $\widehat{\sigma}$ & 1 \\
Dispersion constant $(\mathrm{J})$ & $A$ & $2.0 \times 10^{-21}$ \\
Initial film thickness $(\mu \mathrm{m})$ & $\delta_{i}$ & 0.282 \\
Apparent contact angle $\left({ }^{\circ}\right)$ & $\theta$ & 19.7 \\
Intrinsic interface radius $(\mu \mathrm{m})$ & $R$ & 909 \\
\hline
\end{tabular}

yields the $u$ velocity distribution of uni-directional flow:

$u=\left(d_{x} p / \mu+\bar{\phi}(x)\right)\left(y^{2} / 2-\delta y\right)$

Using this velocity distribution, a new film thickness distribution having the effect of $v$-velocity from SEM solver via the term $\bar{\phi}(x)$, can be obtained. However, a single iteration cycle is not enough to

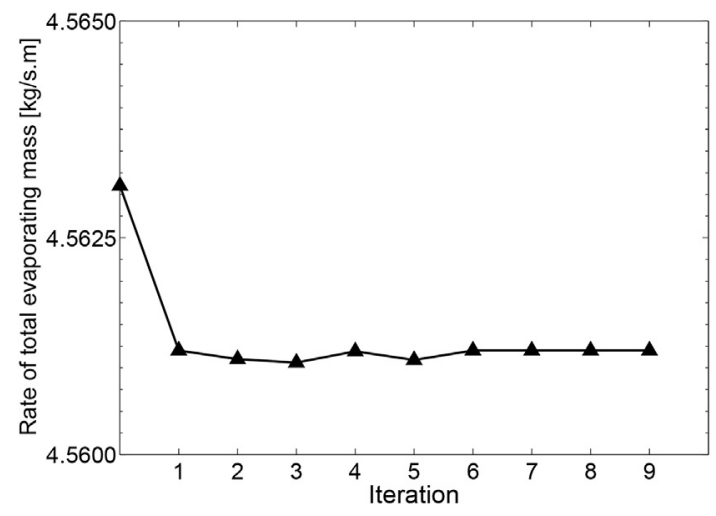

Fig. 3. Change of the total rate of evaporation. 
satisfy the conservation of mass and momentum at the same time. The new film thickness distribution and the new evaporative heat flux distribution as a function of film thickness, are not used as the boundary or boundary condition to the bi-directional flow domain. Then, using the new film thickness and evaporative heat flux distributions, another iteration should be made. After that, unidirectional flow model can be applied again to add the effect of new values of $\bar{\phi}(x)$ to the conservation of mass. This iterative procedure must be repeated till the convergence.

\section{Results and discussion}

The primary aim of the evaporation models is to predict the total rate of evaporation from the thin film region of an evaporating

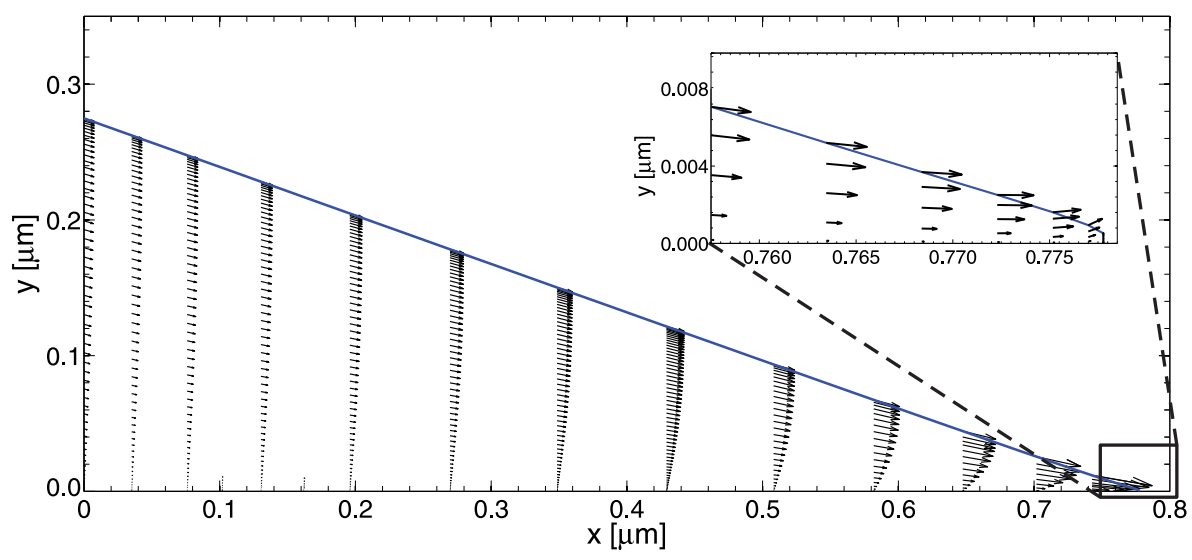

(a)

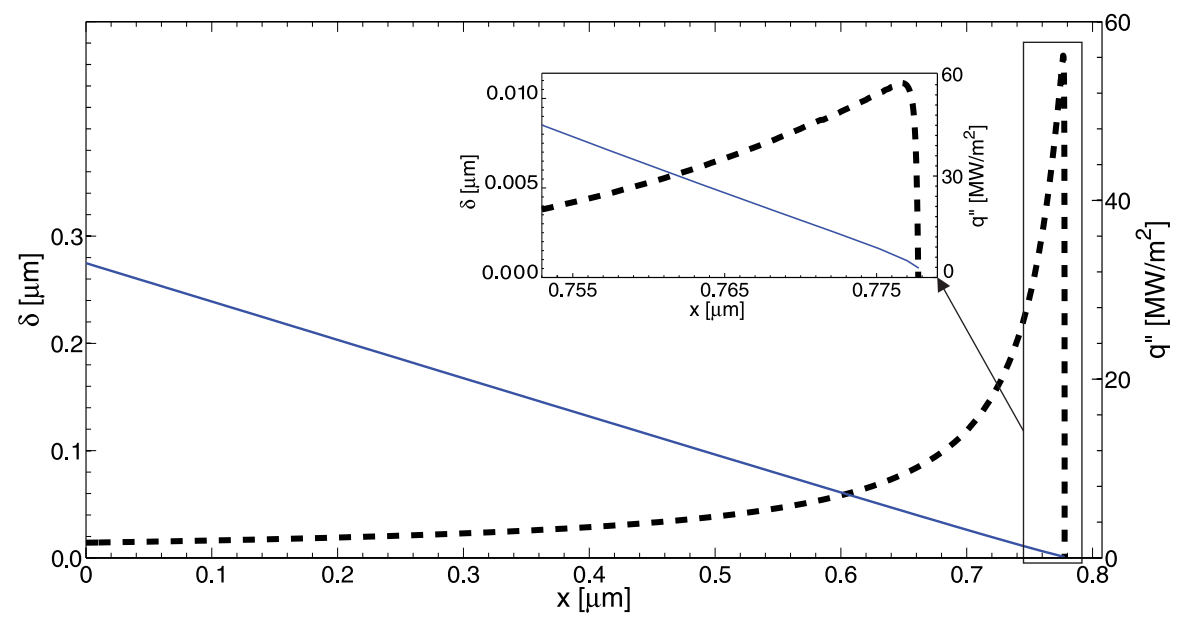

(b)

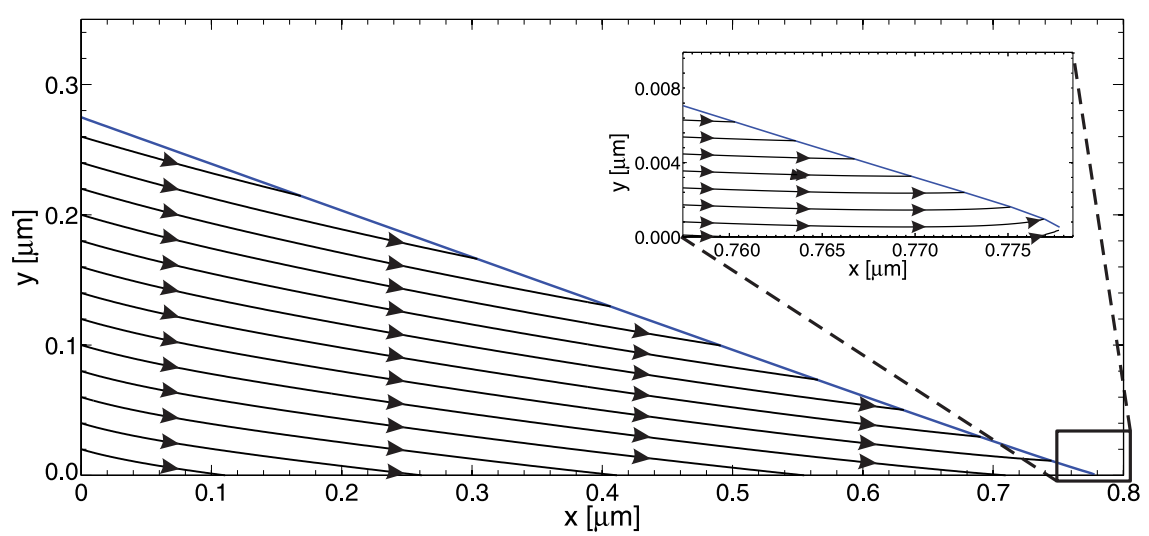

(c)

Fig. 4. (a) Distribution of velocity; (b) Distribution of evaporative heat flux; (c) Streamlines. 
meniscus. Due to the fact that a substantial amount of evaporation takes place within the thin film region, total rate of evaporation in the thin film region determines the general performance of heat removal from the solid substrate on which meniscus is lying. Therefore, the total rate of evaporation is a representative parameter to be used in the convergence decision of the iterative solution process. For the physical example, data of [19] is used, which is the same data set used in the study of Stephan and Busse [5], as summarized in Table 1 . The reason for the use of this data set is that there exists very few studies which provide a complete set of physical input data necessary for a numerical analysis.

The resulting total rate of evaporation at the end of each iteration cycle is plotted in Fig. 3 for nine iterations and it should be noted that each iteration consists of the full computational scheme. It can be seen that numerical convergence is achieved in seven iterations. Besides, there is a deviation between the initial result, calculated using the uni-directional model, and consecutive results, calculated by applying the computational scheme. This trend demonstrates the effect of transverse velocity on the evaporation phenomena.

The resultant distributions of bi-directional flow velocity, evaporative heat flux and the streamlines are given in Fig. 4a to c. The magnitudes of the velocity vectors increase with decreasing film thickness, which is a result of decreasing flow area despite increasing evaporation rates. Fluid continues to accelerate until peak evaporation flux is reached. Due to the sharp increase of disjoining pressure, evaporation is suppressed and eventually vanishes at the contact line where fluid comes to rest. The distance between the maximum evaporation point and the contact line is excessively small. Therefore, flow starts to decelerate before the point of maximum evaporation and stagnates at the contact line. The nature of the evaporation curve at the close proximity of the contact line given in the inset of Fig. $4 \mathrm{~b}$ shows that the apparent discontinuous drop in evaporative flux is actually a continuous, resolved variation. In the neighbourhood of the contact line, the transverse component of the velocity is prominent due to the high rate of evaporation. This can also be seen in Fig. 4c which gives the streamlines of liquid flow. Near the entrance the streamlines are almost parallel to the liquid-vapor interface due to low evaporation rates. As the thickness of the film decreases, the evaporative mass increases resulting in streamlines intersecting the interface at larger angles depicting the transverse component of the velocity. The flow is forced towards the wall due to the contraction of flow area, thus resulting in a negative contribution to transverse velocity, however, the evaporation on the surface forces transverse velocity to be positive, resulting in streamlines intersecting the interface. In the close proximity of contact line the streamlines curve towards the interface due to the high rates of evaporation.

With the application of bi-directional model, the amount of inlet mass flow, the film thickness profile and evaporative heat flux distribution are not affected substantially, which renders the application of bi-directional flow based evaporation model to a practical problem questionable. However, resolving the bidirectional velocity distribution in the thin film region enables the understanding of the underlying physical phenomena. A close inspection of Fig. 5 shows that the effect of the transverse velocity is particularly important near the close proximity of the contact line.

In a recent study, Lim et al. [23] present two-dimensional distributions of velocity and streamlines for a superheat value of $5 \mathrm{~K}$ for an evaporating thin film of ammonia. Although a quantitative comparison with the current study is not possible since far field boundary conditions were not reported explicitly in Ref. [23], qualitatively, distribution of streamlines is in agreement with those of Fig. 4c.

The current problem was formulated and solved for a

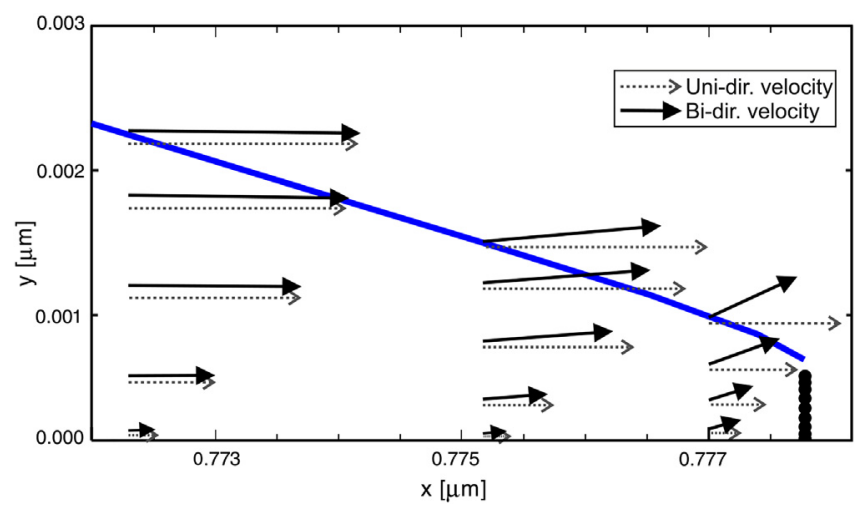

Fig. 5. Velocity distribution comparison of uni-directional and bi-directinol models.

hypothetical perfectly flat surface with no surface roughness or waviness. As the results in Fig. 4a to c shows, the thickness of the liquid film assumes magnitudes of the order of inter-atomic distances near the contact line, rendering the continuum approach questionable. In addition, in manufactured actual surfaces, surface roughness values range between nanometers to micrometers, adding a further complication to the problem. It is, nevertheless, a common practice in the analysis of thin films to use continuum approach despite these shortcomings, therefore, the interpretation of the underlying physics based on these results should be carefully developed.

The fast convergence of the iterative cycle given in Fig. 3, can be attributed to the initial guess, which is very close to the converged result of the iterative cycle. In problems with different geometries and liquids with different physical properties, the effect of the transverse velocity could be more prominent which could affect the rate of convergence of the iterative cycle, whose initial value is obtained from the uni-directional model.

\section{Conclusion}

A solution methodology was developed to enable the solution of steady, 2-D, incompressible conservation of mass and linear momentum equations for the liquid flow in an evaporating thin film. In the solution procedure, a high precision uni-directional solver, a higher order bi-directional spectral element solver and a finite element solver are coupled in the solution of governing equations, without the exclusion of any terms.

The solution of the current 2-D thin film evaporation problem has several challenges. Neither the contact line nor the interface shape is known a priori to the solution. Moreover, the resolution of the interface shape near the contact line is particularly difficult due to rapid changes in the evaporating flux and its effects on the derivatives. The proposed solution algorithm enables the update of both the contact line position and the interface shape within an iteration cycle. Besides, the application of quadruple precision numerics in the uni-directional modeling and the utilization of SEM in the bi-directional modeling enable the calculation of highly accurate derivatives and the quantification of highly resolved transverse velocity in the close vicinity of the contact line.

The current approach may be extended to problems involving a liquid-vapor interface, whose shape is a function of capillary and dispersion pressures. The solution of such problems, using routine techniques currently available in the literature, is not straightforward.

The total evaporating mass obtained from the uni-directional model and the current iterative process are very close, 
nevertheless, the results show the strong transverse motion of the liquid towards the interface near the contact line. In problems with higher contact angles or higher superheats, the effect of bidirectionality is more prominent, and as such, their solution is expected to be more sensitive to the inclusion of the effect of transverse velocity, emphasizing the conservation of momentum in the transverse direction.

\section{Appendix A. The equilibrium pressure difference at the liquid-vapor interface}

\section{Appendix A.1. Clapeyron effect}

Mathematical expression for the Clasius-Clapeyron equation is given in Equation (A.1),

$\frac{d p}{d T}=\frac{h_{l v}}{\Delta v} \frac{1}{T}$

Transition between vapor and liquid phases occurs at temperatures much lower than the critical temperature. In this case, the specific volume of the vapor phase is much larger than that of the liquid phase. Therefore, the difference between the specific volume of the phases can be approximated to the specific volume of vapor phase,

$\Delta v=v_{g}\left(1-\frac{v_{l}}{v_{g}}\right) \approx v_{g}$.

Furthermore, assuming low pressure, equation of state of vapor phase can be approximated by the ideal gas law,

$v_{g}=\frac{R_{u}}{M} \frac{T}{p}$.

With the substitution of Equations (A.2) and (A.3), the ClasiusClapeyron equation for ideal gases becomes as follows,

$\frac{d p}{d T}=\frac{p}{T^{2}} \frac{h_{l v} M}{R_{u}}$.

Equation (A.4), then, is integrated between the states of the bulk vapor and the vapor at an infinitesimal distance above the interface,

$\ln \left(\frac{p_{v, l v}}{p_{v}}\right)=\frac{h_{l v} M}{R_{u}}\left(\frac{1}{T_{v}}-\frac{1}{T_{l v}}\right)$.

Truncation of the Taylor series expansion of the left side of Equation (A.5) yields Equation (A.6):

$\left(\frac{p_{v, l v}}{p_{v}}\right)-1=\frac{h_{l v} M}{R_{u}}\left(\frac{1}{T_{v}}-\frac{1}{T_{l v}}\right)$.

After algebraic manipulations, the difference between the pressure at the interface and vapor can be expressed as a function of superheat,

$p_{v, l v}-p_{v}=\frac{h_{l v} M p_{v}}{R_{u} T_{l v} T_{v}}\left(T_{l v}-T_{v}\right)$.

\section{Appendix A.2. Curvature effect}

The change in vapor pressure due to a curved liquid-vapor interface, is expressed by the Kelvin equation:

$\ln \left(\frac{p_{v, l v}}{p_{v}}\right)=-\frac{M}{\rho_{l} R_{u} T_{l v}} p_{c}$.
When the evaporating film is thin enough, dispersion forces start affecting the interface shape in addition to the capillary force. Disjoining pressure and capillary pressure have additive effects on the film vapor pressure [33]. Therefore, modified Kelvin equation is used in the calculation of vapor pressures of evaporating thin films:

$\ln \left(\frac{p_{v, l v}}{p_{v}}\right)=-\frac{M}{\rho_{l} R_{u} T_{l v}}\left(p_{c}+p_{d}\right)$.

Truncation of the Taylor series expansion of the left side of Equation (A.9) yields:

$\frac{p_{v, l v}}{p_{v}}-1=-\frac{M}{\rho_{l} R_{u} T_{l v}}\left(p_{c}+p_{d}\right)$.

Equation (A.10) can be arranged to express the pressure difference at the interface as a function of capillary and disjoining pressures,

$p_{v, l v}-p_{v}=-\frac{V_{l} p_{v}}{R_{u} T_{l v}}\left(p_{c}+p_{d}\right)$

where $V_{l}$ is the molar volume of the liquid.

\section{Appendix B. Application of SEM to equations (22a) and (22b)}

Appendix B.1. Weak formulation

The weak formulation of the equations is a variational statement of the problem. To achieve a weak form of a differential equation, the equation is multiplied by a test function and integrated over the domain. The selection of the test functions can change according to the method preferred. When the dependent variable of the differential equation is approximated by trial functions, the result of integration is not exactly zero but equals to a residual. Then, the aim is to minimize the error between the actual and approximate solutions.

For convenience, Equations (22a) and (22b) are written in vector form in the rest of the weak formulation:

$\mathbf{u} \cdot \nabla \mathbf{u}=\nabla \cdot \overline{\bar{\sigma}}+\mathbf{F}$

where the vector, $\mathbf{F}$, has the following form:

$\mathbf{F}=[\nabla p]^{T}$.

The space of test functions, $V$, is defined on the boundaries as follows:

$V=\left\{\mathbf{v} \in E(\Omega)|\quad \mathbf{v}|_{x=0}=\left.\mathbf{v}\right|_{x=\ell^{*}}=\left.\mathbf{v}\right|_{y=0}=0 ;\left.\quad \mathbf{v} \cdot \mathbf{n}\right|_{y=\delta}=0\right\}$,

where $\mathbf{v}$ is test function and $E(\Omega)$ is energy space.

Equation (B.1) is projected onto the space of test functions. The result of the integration is given below, in index notation:

$\iint_{\Omega} v_{j} u_{i} \partial_{i} u_{j} d \Omega=\iint_{\Omega} v_{j} \partial_{i} \sigma_{i j} d \Omega+\iint_{\Omega} v_{j} F_{j} d \Omega$.

The first term on the right hand side of Equation (B.4) can be rewritten applying the chain rule,

$\iint_{\Omega} v_{j} u_{i} \partial_{i} u_{j} d \Omega=\iint_{\Omega}\left[\partial_{i}\left(v_{j} \sigma_{i j}\right)-\sigma_{i j} \partial_{i} v_{j}\right] d \Omega+\iint_{\Omega} v_{j} F_{j} d \Omega$

Considering only two-dimensional vector fields, divergence theorem is applied to the first term in the integrand of the first term on the right hand side of Equation (B.5): 


$$
\iint_{\Omega} v_{j} u_{i} \partial_{i} u_{j} d \Omega=\int_{\partial \Omega} n_{i} \cdot\left(v_{j} \sigma_{i j}\right) d s-\iint_{\Omega} \sigma_{i j} \partial_{i} v_{j} d \Omega+\iint_{\Omega} v_{j} F_{j} d \Omega .
$$

The first term on the right hand side of Equation (B.6) is equal to zero due to the definition of test function on the boundaries. Then, the weak formulation of the problem yields Equation (B.7), which is solved using trial functions for approximation,

$\iint_{\Omega} v_{j} u_{i} \partial_{i} u_{j} d \Omega=-\iint_{\Omega} \sigma_{i j} \partial_{i} v_{j} d \Omega+\iint_{\Omega} v_{j} F_{j} d \Omega$

Equation (B.7) can also be expressed in vector-operator notation:

$$
\iint_{\Omega}(\mathbf{v} \otimes \mathbf{u}): \nabla \mathbf{u} d \Omega=-\iint_{\Omega} \overline{\bar{\sigma}}: \nabla \mathbf{v} d \Omega+\iint_{\Omega} \mathbf{v} \cdot \mathbf{F} d \Omega .
$$

\section{Appendix B.2. Mapping}

In order to perform the numerical differentiations and integrations using Gauss-Lobatto-Legendre (GLL) nodes, the problem needs to be transformed to a standard domain. Therefore, actual irregular physical domain, $\Omega$, is mapped to the unit square, $\Omega_{s t}$.
The calculation of mixed derivatives is necessary to evaluate the numerical differentiation operations in Equation (B.8). Mixed derivatives of transformation appears as in the form of Equations (B.12a) to (B.12d):

$\partial_{x} S=2 / \ell^{*}$

$\partial_{y} s=0$,

$\partial_{X} t=-\left(2 / \ell^{*}\right)(1+t)\left(\delta^{\prime} / \delta\right)$,

$\partial_{y} t=2 / \delta$.

The gradient operator, then, becomes,

$$
\nabla=\left[\begin{array}{c}
\partial_{x} \\
\partial_{y}
\end{array}\right]=\left[\begin{array}{c}
\left(2 / \ell^{*}\right) \partial_{s}-\left(2 / \ell^{*}\right)(1+t)\left(\delta^{\prime} / \delta\right) \partial_{t} \\
(2 / \delta) \partial_{t}
\end{array}\right]
$$

\section{Appendix B.3. Discretization}

Dependent variable of the problem, which is the velocity $\mathbf{u}$, in Equations (B.7) and (B.8) of the current study, is discretized by using polynomials. In Galerkin approach, polynomial expansion is
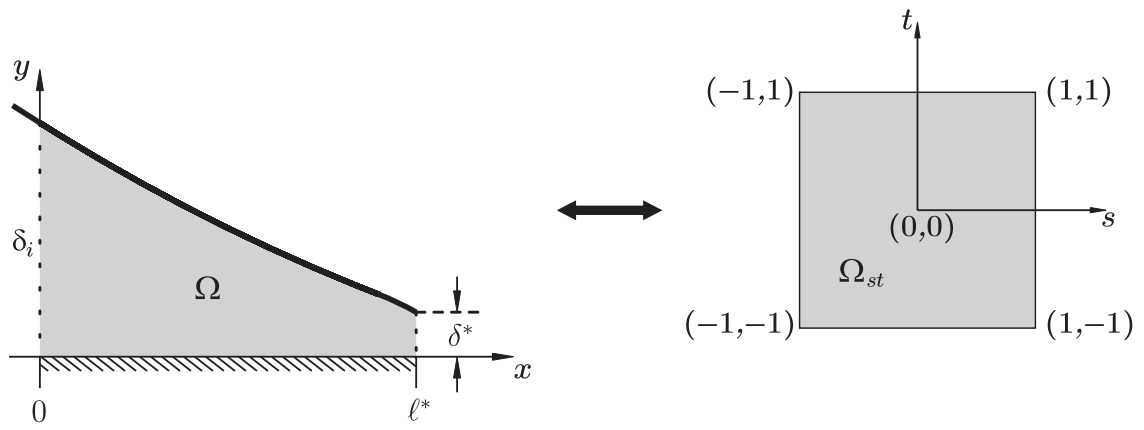

Figure B.6. Mapping of the physical domain to a standard domain.

The relation between actual and standard coordinates is constructed by using the mapping functions, $X_{1}$ and $X_{2}$ :

$x=X_{1}(s, t)$

$y=X_{2}(s, t)$.

Mapping functions, on the other hand, are constructed using linear blending [34]. In this problem, mapping functions for the longitudinal and transverse coordinates are found as follows,

$X_{1}(s, t)=\frac{1+s}{2} l^{*}$,

$X_{2}(s, t)=\delta\left(\frac{1+s}{2} l^{*}\right) \frac{1+t}{2}$

where $-1 \leq s, t \leq 1$ and $\delta$ is a function of $s$. In the rest of the derivations, $\delta$ will be used without its argument for clarity.

The Jacobian of transformation is determined in Equation (B.11),

$|J|=\left|\frac{\partial(s, t)}{\partial(x, y)}\right|=\left|\left(\begin{array}{cc}\ell^{*} / 2 & 0 \\ \delta^{\prime}(1+t)\left(\ell^{*} / 4\right) & \delta / 2\end{array}\right)\right|=\delta\left(\ell^{*} / 4\right)$. used for both trial space, $U$, and test space, $V$. Polynomial approximation of the dependent variable is shown in Equation (B.14):

$\left[\begin{array}{l}u \\ v\end{array}\right](x, y) \cong \sum_{p=0}^{M} \sum_{q=0}^{N}\left[\begin{array}{l}u_{p q} \\ v_{p q}\end{array}\right] E_{p}(s) E_{q}(t)$

where $€$ denotes Lagrange polynomial interpolant through GaussLobatto-Legendre (GLL) points, $\left\{s_{p}, t_{q}\right\}$, typically,

$\iota_{p}(s)=\prod_{m=0, m \neq p}^{M} \frac{s-s_{m}}{s_{p}-s_{m}}$ and $\iota_{\mathrm{q}}(\mathrm{t})=\prod_{\mathrm{n}=0, \mathrm{n} \neq \mathrm{q}}^{\mathrm{N}} \frac{\mathrm{t}-\mathrm{t}_{\mathrm{n}}}{\mathrm{t}_{\mathrm{q}}-\mathrm{t}_{\mathrm{n}}}$

where $\left[\begin{array}{c}u_{p q} \\ v_{p q}\end{array}\right]=\left[\begin{array}{l}u \\ v\end{array}\right]\left(X_{1}\left(s_{p}, t_{q}\right), X_{2}\left(s_{p}, t_{q}\right)\right)$.

At the GLL nodes, Lagrange interpolants, Equation (B.15), exhibit Kronecker-Delta property,

$\iota_{p}\left(s_{m}\right)=\delta_{p m}$

$\iota_{q}\left(t_{n}\right)=\delta_{q n}$

with $-1=s_{0}<\ldots<s_{M}=1$ and $-1=t_{0}<\ldots<t_{N}=1$.

GLL nodes together with the associated weights $\left\{w_{m}, w_{n}\right\}$ 
provide high accuracy quadrature approximation for integrals. A representative integration for the longitudinal velocity on the physical domain is performed in the following equations.

First, actual physical domain is transformed to the standard domain:

$\iint_{\Omega} u(x, y) d \Omega=\iint_{\Omega_{s t}} u(x(s, t), y(s, t)) J d s d t$.

Second, discretization of the dependent variable is performed:

$\iint_{\Omega} u(x, y) d \Omega \cong \sum_{p=0}^{M} \sum_{q=0}^{N} u_{p q} \iint_{\Omega_{s t}} J t_{p}(s) E_{p}(t) d s d t$.

Next, numerical integration is applied with the associated weights $\left\{w_{k}, w_{l}\right\}$ :

$\iint_{\Omega} u(x, y) d \Omega \cong \sum_{p=0}^{M} \sum_{q=0}^{N} u_{p q}\left(\sum_{k=0}^{M} \sum_{l=0}^{N} J\left(s_{k}, t_{l}\right) E_{p}\left(s_{k}\right) E_{q}\left(t_{l}\right) w_{k} w_{l}\right)$.

Then, Kronecker-Delta property of the Lagrange interpolants is used:

$\iint_{\Omega} u(x, y) d \Omega \cong \sum_{p=0}^{M} \sum_{q=0}^{N} u_{p q}\left(\sum_{k=0}^{M} \sum_{l=0}^{N} J\left(s_{k}, t_{l}\right) \delta_{p k} \delta_{q l} w_{k} w_{l}\right)$.

The final, transformed and integrated form of the term can be seen in Equation (B.21):

$\iint_{\Omega} u(x, y) d \Omega \cong \sum_{k=0}^{M} \sum_{l=0}^{N} u_{k l} J_{k l} w_{k} w_{l}$

where $J_{k l}=J\left(X_{1}\left(s_{k}, t_{l}\right), X_{2}\left(s_{k}, t_{l}\right)\right)$.

Discretized formulation yields a definite set of equations. The number of equations is equal to the number of GLL nodes used in the domain. In this problem, $61 \times 61$ nodes are used to discretize the domain and one single element is used to represent the entire domain. Thus, the polynomials which approximate the trial and test space, are of $60^{\text {th }}$ degree.

\section{Appendix B.4. Boundary conditions}

Boundary conditions were given in Equations (23a)-(23d) for the actual physical domain. These boundary conditions should also be transformed and discretized for a standard domain on which numerical calculation is performed.

Equation (23a), which states zero velocities at the wall, is result of the no-slip condition. Therefore, velocities defined at the nodes of this boundary are zero:

$u_{p 0}=v_{p 0}=0$ for $p=0,1, \ldots, M$

Equation (23b) shows the initial velocity distributions, $u_{0}$ and $v_{0}$. They are assumed to have parabolic profiles. Their magnitudes are specified to satisfy the amount of inlet mass flow. The amount of inlet mass flow, on the other hand, cannot be known a priori because it must be equal to the total evaporated liquid which is one of the results of the complete analysis. Thus, the inlet mass flow, and the initial velocity distributions are subjected to change at every iterative step. Discretization of the initial velocities at this boundary are given in Equation (B.23):

$$
\begin{aligned}
u_{0 q} & =u_{0}\left(X_{2}\left(s_{0}, t_{q}\right)\right) \text { and } v_{0 q}=v_{0}\left(X_{2}\left(s_{0}, t_{q}\right)\right) \text { for } q \\
& =0,1, \ldots, N .
\end{aligned}
$$

Equation (23c) states the relation between evaporative mass flux from the liquid-vapor interface and fluid velocities at the interface. As in the case of initial velocities, evaporative mass flux is also subject to change because the distribution of evaporative mass flux is a result of complete analysis and cannot be known a priori. Before giving the discretization of the boundary condition at interface, unit normal vector to the surface must be defined:

$\mathbf{n}=\frac{-\delta^{\prime}}{\sqrt{1+\left(\delta^{\prime}\right)^{2}}} \mathbf{i}+\frac{1}{\sqrt{1+\left(\delta^{\prime}\right)^{2}}} \mathbf{j}$

After the multiplication of unit normal vector with velocity vector, Equation (23c) can be rewritten as shown by Equation (B.25):

$-\delta^{\prime} u+v=\frac{\sqrt{1+\left(\delta^{\prime}\right)^{2}}}{\rho} m_{\text {evap }}^{\prime \prime}$

The boundary condition of surface velocities at the free surface can also be expressed as follows:

$$
\begin{aligned}
-u_{p N} \delta^{\prime}\left(s_{p}\right)+v_{p N} & =\frac{\sqrt{1+\left(\delta^{\prime}\left(s_{p}\right)\right)^{2}}}{\rho} m^{\prime \prime} \text { evap }\left(s_{p}\right) \text { for } p \\
& =0,1, \ldots, M .
\end{aligned}
$$

Equation (23d) is the boundary condition at the contact line where liquid comes to rest. Therefore, the velocities at the nodes of this boundary are zero,

$u_{M q}=v_{M q}=0$ for $q=0,1, \ldots, N$.

For the homogeneous boundary conditions, test functions, $v$, are discretized in the same way and after implementing boundary conditions the resultant set of equations is solved using the MATLAB software. In the numerical solution procedure, Picard iteration is used to handle the non-linear terms of the Navier-Stokes equation.

\section{References}

[1] Plawsky JL, Fedorov AG, Garimella SV, Ma HB, Maroo SC, Chen L, et al. Nanoand microstructures for thin-film evaporation a review. Nanosc Microsc Therm 2014:18(3):251-69.

[2] Nikolayev VS. Dynamics of the triple contact line on a nonisothermal heater at partial wetting. Phys Fluids 2010;22(8):082105.

[3] Wayner Jr PC, Kao YK, Lacroix LV. The interline heat-transfer coefficient of an evaporating wetting film. Int J Heat Mass Tran 1976;19:487-92.

[4] Sujanani M, Wayner Jr PC. Microcomputer-enhanced optical investigation of transport processes with phase change in near-equilibrium thin liquid films. Coll Interf Sci 1991;2:472-88.

[5] Stephan PC, Busse CA. Analysis of the heat transfer coefficient of grooved heat pipe evaporator walls. Int J Heat Mass Tran 1992;35(2):383-91.

[6] Dasgupta S, Schonberg JA, Kim IY, Wayner Jr PC. Use of the augmented younglaplace equation to model equilibrium and evaporating extended menisci. J Coll Interf Sci 1993;157:332-42.

[7] Dasgupta S, Schonberg JA, Wayner Jr PC. Investigation of an evaporating extended meniscus based on the augmented young-laplace equation. J Heat Transf 1993; 115:201-8.

[8] Dasgupta S, Kim IY, Wayner Jr PC. Use of the kelvin-clapeyron equation to model an evaporating curved microfilm. J Heat Transf 1994:116:1007-15.

[9] Schonberg JA, Dasgupta S, Wayner Jr PC. An augmented young-laplace model of an evaporating meniscus in a microchannel with high heat flux. Exp Therm Fluid Sci 1995;10:163-70.

[10] Hocking LM. On contact angles in evaporating liquids. Phys Fluids 1995;7(12): 2950-5.

[11] Morris SJS. A phenomenological model for the contact region of an 
evaporating meniscus on a superheated slab. J Fluid Mech 2000;411:59-89.

[12] Ajaev VS, Homsy GM. Three-dimensional steady vapor bubbles in rectangular microchannels. J Coll Interf Sci 2001;244(1):180-9.

[13] Wang H, Garimella SV, Murthy JY. Characteristics of an evaporating thin film in a microchannel. Int J Heat Mass Tran 2007;50:3933-42.

[14] Do KH, Kim SJ, Garimella SV. A mathematical model for analyzing the thermal characteristics of a flat micro heat pipe with a grooved wick. Int J Heat Mass Tran 2008;51:4637-50.

[15] Bertossi R, Lataoui Z, Ayel V, Romestant C, Bertin Y. Modeling of thin liquid film in grooved heat pipes. Numer Heat Transf A 2009;55:1075-95.

[16] Du S, Zhao YH. New boundary conditions for the evaporating thin-film model in a rectangular micro channel. Int J Heat Mass Tran 2011;54:3694-701.

[17] Hanchak MS, Vangsness MD, Byrd LW, Ervin JS. Thin film evaporation of noctane on silicon: experiments and theory. Int J Heat Mass Tran 2014;75: 196-206.

[18] Kou ZH, Lv HT, Zeng W, Bai ML, Lv JZ. Comparison of different analytical models for heat and mass transfer characteristics of an evaporating meniscus in a micro-channel. Int Commun Heat Mass 2015;63:49-53.

[19] Akkuş Y, Dursunkaya Z. A new approach to thin film evaporation modeling. Int J Heat Mass Tran 2016:101:742-8.

[20] Anderson DM, Davis SH. The spreading of volatile liquid droplets on heated surfaces. Phys Fluids 1995;7(2):248-65.

[21] Tsoumpas Y, Dehaeck S, Rednikov A, Colinet P. Effect of marangoni flows on the shape of thin sessile droplets evaporating into air. Langmuir 2015;31(49): $13334-40$.

[22] Lim E, Hung YM. Thermophysical phenomena of working fluids of thermocapillary convection in evaporating thin liquid films. Int Commun Heat Mass 2015;66:203-11.
[23] Lim E, Hung YM, Tan BT. A hydrodynamic analysis of thermocapillary convection in evaporating thin liquid films. Int J Heat Mass Transf 2017;108: 1103-14.

[24] Ren W, Hu D, Weinan E. Continuum models for the contact line problem. Phys Fluids 2010;22(10):102103.

[25] G. Ball, J. Polansky, T. Kaya, Investigation of particular features of the numerical solution of an evaporating thin film in a channel, FHMT 4(1).

[26] Wee SK, Kihm KD, Hallinan KP. Effects of the liquid polarity and the wall slip on the heat and mass transport characteristics of the micro-scale evaporating transition film. Int J Heat Mass Tran 2005;48:265-78.

[27] Benselama AM, Harmand S, Sefiane K. Thermocapillary effects on steadily evaporating contact line: a perturbative local analysis. Phys Fluids 2012;24(7): 072105.

[28] Moosman S, Homsy GM. Evaporating menisci of wetting fluids. J Coll Interf Sci $1980 ; 73: 212-23$.

[29] Slattery JC, Sagis L, Oh ES. Interfacial transport phenomena. Springer Science \& Business Media; 2007.

[30] Deryagin BV, Churaev NV. Definition of disjoining pressure and its importance in equilibrium and flow of thin-films. Colloid J USSR 1976;38(3):402-10.

[31] Kucherov RJ, Rikenglaz LE. The measurement of the condensation coefficient Dokl Akad Nauk SSSR 1960;133(5):1130-1.

[32] Schrage RW. A theoretical study of interphase mass transfer. New York: Columbia University Press; 1953.

[33] Padday JF. Cohesive properties of thin films on liquids adhering to a solid surface. Special Discuss Faraday Soc 1970;1:64-74.

[34] Gordon WJ, Hall CA. Transfinite element methods: blending-function interpolation over arbitrary curved element domains. Numer Math 1973;21: 109-209. 